\title{
Research Article \\ Remarks on Cone Metric Spaces and Fixed Point Theorems of Contractive Mappings
}

\author{
Mohamed A. Khamsi ${ }^{1,2}$ \\ ${ }^{1}$ Department of Mathematical Science, The University of Texas at El Paso, El Paso, TX 79968, USA \\ 2 Department of Mathematics and Statistics, King Fahd University of Petroleum E Minerals, P.O. Box 411, \\ Dhahran 31261, Saudi Arabia
}

Correspondence should be addressed to Mohamed A. Khamsi, mohamed@utep.edu

Received 20 March 2010; Accepted 4 May 2010

Academic Editor: W. A. Kirk

Copyright @ 2010 Mohamed A. Khamsi. This is an open access article distributed under the Creative Commons Attribution License, which permits unrestricted use, distribution, and reproduction in any medium, provided the original work is properly cited.

We discuss the newly introduced concept of cone metric spaces. We also discuss the fixed point existence results of contractive mappings defined on such metric spaces. In particular, we show that most of the new results are merely copies of the classical ones.

\section{Introduction}

Cone metric spaces were introduced in [1]. A similar notion was also considered by Rzepecki in [2]. After carefully defining convergence and completeness in cone metric spaces, the authors proved some fixed point theorems of contractive mappings. Recently, more fixed point results in cone metric spaces appeared in [3-8]. Topological questions in cone metric spaces were studied in [6] where it was proved that every cone metric space is first countable topological space. Hence, continuity is equivalent to sequential continuity and compactness is equivalent to sequential compactness. It is worth mentioning the pioneering work of Quilliot [9] who introduced the concept of generalized metric spaces. His approach was very successful and used by many (see references in [10]). It is our belief that cone metric spaces are a special case of generalized metric spaces. In this work, we introduce a metric type structure in cone metric spaces and show that classical proofs do carry almost identically in these metric spaces. This approach suggest that any extension of known fixed point result to cone metric spaces is redundant. Moreover the underlying Banach space and the associated cone subset are not necessary.

For more on metric fixed point theory, the reader may consult the book [11]. 


\section{Basic Definitions and Results}

First let us start by making some basic definitions.

Definition 2.1. Let $E$ be a real Banach space with norm $\|\cdot\|$ and $P$ a subset of $E$. Then $P$ is called a cone if and only if

(1) $P$ is closed, nonempty, and $P \neq\{\theta\}$, where $\theta$ is the zero vector in $E$;

(2) if $a, b \geq 0$, and $x, y \in P$, then $a x+b y \in P$;

(3) if $x \in P$ and $-x \in P$, then $x=\theta$. by

Given a cone $P$ in a Banach space $E$, we define a partial ordering $\preceq$ with respect to $P$

$$
x \preceq y \Longleftrightarrow y-x \in P .
$$

We also write $x<y$ whenever $x \leq y$ and $x \neq y$, while $x \ll y$ will stand for $y-x \in \operatorname{Int}(P)$ (where $\operatorname{Int}(P)$ designate the interior of $P$ ). The cone $P$ is called normal if there is a number $K>0$, such that for all $x, y \in E$, we have

$$
\theta \preceq x \preceq y \Longrightarrow\|x\| \leq K\|y\|
$$

The least positive number satisfying this inequality is called the normal constant of $P$. The cone $P$ is called regular if every increasing sequence which is bounded from above is convergent. Equivalently the cone $P$ is called regular if every decreasing sequence which is bounded from below is convergent. Regular cones are normal and there exist normal cones which are not regular.

Throughout the Banach space $E$ and the cone $P$ will be omitted.

Definition 2.2. A cone metric space is an ordered pair $(X, d)$, where $X$ is any set and $d: X \times$ $X \rightarrow E$ is a mapping satisfying

(1) $d(x, y) \in P$, that is, $\theta \leq d(x, y)$, for all $x, y \in X$, and $d(x, y)=\theta$ if and only if $x=y$;

(2) $d(x, y)=d(y, x)$ for all $x, y \in X$;

(3) $d(x, y) \preceq d(x, z)+d(z, y)$, for all $x, y, z \in X$.

Convergence is defined as follows.

Definition 2.3. Let $(X, d)$ be a cone metric space, let $\left\{x_{n}\right\}$ be a sequence in $X$ and $x \in X$. If for any $c \in P$ with $c \gg \theta$, there is $N \geq 1$ such that for all $n \geq N, d\left(x_{n}, x\right) \ll c$, then $\left\{x_{n}\right\}$ is said to be convergent. We will say $\left\{x_{n}\right\}$ converges to $x$ and write $\lim _{n \rightarrow \infty} x_{n}=x$.

It is easy to show that the limit of a convergent sequence is unique. Cauchy sequences and completeness are defined by

Definition 2.4. Let $(X, d)$ be a cone metric space, $\left\{x_{n}\right\}$ be a sequence in $X$. If for any $c \in P$ with $c \gg \theta$, there is $N \geq 1$ such that for all $n, m \geq N, d\left(x_{n}, x_{m}\right) \ll c$, then $\left\{x_{n}\right\}$ is called Cauchy sequence. If every Cauchy sequence is convergent in $X$, then $X$ is called a complete cone metric space. 
The basic properties of convergent and Cauchy sequences may be found at [1]. In fact the properties and their proofs are identical to the classical metric ones. Since this work concerns the fixed point property of mappings, we will need the following property.

Definition 2.5. Let $(X, d)$ be a cone metric space. A mapping $T: X \rightarrow X$ is called Lipschitzian if there exists $k \in \mathbb{R}$ such that

$$
d(T x, T y) \leq k d(x, y)
$$

for all $x, y \in X$. The smallest constant $k$ which satisfies the above inequality is called the Lipschitz constant of $T$, denoted $\operatorname{Lip}(T)$. In particular $T$ is a contraction if $\operatorname{Lip}(T) \in[0,1)$.

As we mentioned earlier cone metric spaces have a metric type structure. Indeed we have the following result.

Theorem 2.6. Let $(X, d)$ be a metric cone over the Banach space $E$ with the cone $P$ which is normal with the normal constant $K$. The mapping $D: X \times X \rightarrow[0, \infty)$ defined by $D(x, y)=\|d(x, y)\|$ satisfies the following properties:

(1) $D(x, y)=0$ if and only if $x=y$;

(2) $D(x, y)=D(y, x)$, for any $x, y \in X$;

(3) $D(x, y) \leq K\left(D\left(x, z_{1}\right)+D\left(z_{1}, z_{2}\right)+\cdots+D\left(z_{n}, y\right)\right)$, for any points $x, y, z_{i} \in X, i=$ $1,2, \ldots, n$.

Proof. The proofs of (1) and (2) are easy and left to the reader. In order to prove (3), let $x, y, z_{1}, \ldots, z_{n}$ be any points in $X$. Using the triangle inequality satisfied by $d$, we get

$$
d(x, y) \leq d\left(x, z_{1}\right)+d\left(z_{1}, z_{2}\right)+\cdots+d\left(z_{n}, y\right) .
$$

Since $P$ is normal with constant $K$ we get

$$
\|d(x, y)\| \leq K\left\|d\left(x, z_{1}\right)+d\left(z_{1}, z_{2}\right)+\cdots+d\left(z_{n}, y\right)\right\|,
$$

which implies

$$
\|d(x, y)\| \leq K\left(\left\|d\left(x, z_{1}\right)\right\|+\left\|d\left(z_{1}, z_{2}\right)\right\|+\cdots+\left\|d\left(z_{n}, y\right)\right\|\right) .
$$

This completes the proof of the theorem.

Note that the property (3) is discouraging since it does not give the classical triangle inequality satisfied by a distance. But there are many examples where the triangle inequality fails (see, e.g., [12]).

The above result suggest the following definition. 
Definition 2.7. Let $X$ be a set. Let $D: X \times X \rightarrow[0, \infty)$ be a function which satisfies

(1) $D(x, y)=0$ if and only if $x=y$;

(2) $D(x, y)=D(y, x)$, for any $x, y \in X$;

(3) $D(x, y) \leq K\left(D\left(x, z_{1}\right)+D\left(z_{1}, z_{2}\right)+\cdots+D\left(z_{n}, y\right)\right)$, for any points $x, y, z_{i} \in X, i=$ $1,2, \ldots, n$, for some constant $K>0$.

The pair $(X, D)$ is called a metric type space.

Similarly we define convergence and completeness in metric type spaces.

Definition 2.8. Let $(X, D)$ be a metric type space.

(1) The sequence $\left\{x_{n}\right\}$ converges to $x \in X$ if and only if $\lim _{n \rightarrow \infty} D\left(x_{n}, x\right)=0$.

(2) The sequence $\left\{x_{n}\right\}$ is Cauchy if and only if $\lim _{n, m \rightarrow \infty} D\left(x_{n}, x_{m}\right)=0$.

$(X, D)$ is complete if and only if any Cauchy sequence in $X$ is convergent.

\section{Some Fixed Point Results}

Let $T: X \rightarrow X$ be a map. $T$ is called Lipschitzian if there exists a constant $\lambda \geq 0$ such that

$$
D(T x, T y) \leq \lambda D(x, y)
$$

for any $x, y \in X$. The smallest constant $\lambda$ will be denoted $\operatorname{Lip}(T)$.

Theorem 3.1. Let $(X, D)$ be a complete metric type space. Let $T: X \rightarrow X$ be a map such $T^{n}$ is Lipschitzian for all $n \geq 0$ and that $\sum_{n=0}^{\infty} \operatorname{Lip}\left(T^{n}\right)<\infty$. Then $T$ has a unique fixed point $\omega \in X$. Moreover for any $x \in X$, the orbit $\left\{T^{n} x\right\}$ converges to $\omega$.

Proof. Let $x \in X$. For any $n, h \geq 0$, we have

$$
D\left(T^{n+h} x, T^{n} x\right) \leq \operatorname{Lip}\left(T^{n}\right) D\left(T^{h} x, x\right) \leq K \operatorname{Lip}\left(T^{n}\right) \sum_{i=0}^{h-1} D\left(T^{i+1} x, T^{i} x\right) .
$$

Hence

$$
D\left(T^{n+h} x, T^{n} x\right) \leq K \operatorname{Lip}\left(T^{n}\right)\left(\sum_{i=0}^{h-1} \operatorname{Lip}\left(T^{i}\right)\right) D(x, T x) .
$$

Since $\sum_{n=0}^{\infty} \operatorname{Lip}\left(T^{n}\right)$ is convergent, then $\lim _{n \rightarrow \infty} \operatorname{Lip}\left(T^{n}\right)=0$. This forces $\left\{T^{n} x\right\}$ to be a Cauchy sequence. Since $X$ is complete, then $\left\{T^{n} x\right\}$ converges to some point $\omega(x)$. First let us show that $\omega(x)$ is a fixed point of $T$. Since

$$
\begin{aligned}
D\left(T^{n-1} x, \omega(x)\right) & \leq K\left(D\left(T^{n-1} x, T^{n} x\right)+D\left(T^{n} x, \omega(x)\right)\right) \\
& \leq K\left(\operatorname{Lip}\left(T^{n-1}\right) D(x, T x)+D\left(T^{n} x, \omega(x)\right)\right)
\end{aligned}
$$


we get

$$
\begin{aligned}
D(\omega(x), T \omega(x)) & \leq K\left(D\left(\omega(x), T^{n} x\right)+D\left(T^{n} x, T \omega(x)\right)\right) \\
& \leq K\left((1+K \operatorname{Lip}(T)) D\left(\omega(x), T^{n} x\right)+K \operatorname{Lip}(T) \operatorname{Lip}\left(T^{n-1}\right) D(x, T x)\right) .
\end{aligned}
$$

If we let $n \rightarrow \infty$, we get $D(\omega(x), T \omega(x))=0$, or $T \omega(x)=\omega(x)$. Next we show that $T$ has at most one fixed point. Indeed let $\omega_{1}$ and $\omega_{2}$ be two fixed points of $T$. Then we have

$$
D\left(\omega_{1}, \omega_{2}\right)=D\left(T^{n} \omega_{1}, T^{n} \omega_{2}\right) \leq \operatorname{Lip}\left(T^{n}\right) D\left(\omega_{1}, \omega_{2}\right)
$$

for any $n \geq 1$. Since $\lim _{n \rightarrow \infty} \operatorname{Lip}\left(T^{n}\right)=0$, we get $D\left(\omega_{1}, \omega_{2}\right)=0$, or $\omega_{1}=\omega_{2}$. Therefore we have $\omega(x)=\omega(y)$ for any $x, y \in X$, which completes the proof of the theorem.

The condition $\sum_{n=0}^{\infty} \operatorname{Lip}\left(T^{n}\right)<\infty$ is needed because of the condition (3) satisfied by $D$. In fact a more natural condition should be

(3') $D(x, y) \leq K(D(x, z)+D(z, y))$, for any points $x, y, z \in X$, for some constant $K>0$. An example of such $D$ satisfying $\left(3^{\prime}\right)$ is given below.

Example 3.2. Let $X$ be the set of Lebesgue measurable functions on $[0,1]$ such that

$$
\int_{0}^{1}|f(x)|^{2} d x<\infty
$$

Define $D: X \times X \rightarrow[0, \infty)$ by

$$
D(f, g)=\int_{0}^{1}|f(x)-g(x)|^{2} d x
$$

Then $D$ satisfies the following properties:

(1) $D(f, g)=0$ if and only if $f=g$;

(2) $D(f, g)=D(g, f)$, for any $f, g \in X$;

(3') $D(f, g) \leq 2(D(f, h)+D(h, g))$, for any points $f, g, h \in X$.

In the next result we consider the case of metric type spaces $(X, D)$ when $D$ satisfies $\left(3^{\prime}\right)$. Recall that a subset $Y$ of $X$ is said to be bounded whenever $\sup \{D(x, y) ; x, y \in Y\}<\infty$.

Theorem 3.3. Let $(X, D)$ be a complete metric type space, where $D$ satisfies ( $\left.3^{\prime}\right)$ instead of (3). Let $T: X \rightarrow X$ be a map such that $T^{n}$ is Lipschitzian for any $n \geq 0$ and $\lim _{n \rightarrow \infty} \operatorname{Lip}\left(T^{n}\right)=0$. Then $T$ has a unique fixed point if and only if there exists a bounded orbit. Moreover if $T$ has a fixed point $\omega$, then for any $x \in X$, the orbit $\left\{T^{n} x\right\}$ converges to $\omega$. 
Proof. Clearly if $T$ has a fixed point, then its orbit is bounded. Conversely let $x \in \mathrm{X}$ such that $\left\{T^{n} x\right\}$ is bounded, that is, there exists $c \geq 0$ such that $D\left(T^{n} x, T^{m} x\right) \leq c$, for any $n, m \geq 0$. Let $n, h \geq 0$, we have

$$
D\left(T^{n+h} x, T^{n} x\right) \leq \operatorname{Lip}\left(T^{n}\right) D\left(T^{h} x, x\right) \leq \operatorname{Lip}\left(T^{n}\right) c
$$

Since $\lim _{n \rightarrow \infty} \operatorname{Lip}\left(T^{n}\right)=0$, then $\left\{T^{n} x\right\}$ is a Cauchy sequence. Hence $\left\{T^{n} x\right\}$ converges to some point $\omega(x)$ since $X$ is complete. The remaining part of the proof follows the same as in the previous theorem.

The connection between the above results and the main theorems of [1] are given in the following corollary.

Corollary 3.4. Let $(X, d)$ be a metric cone over the Banach space $E$ with the cone $P$ which is normal with the normal constant $K$. Consider $D: X \times X \rightarrow[0, \infty)$ defined by $D(x, y)=\|d(x, y)\|$. Let $T: X \rightarrow X$ be a contraction with constant $k<1$. Then

$$
D\left(T^{n} x, T^{n} y\right) \leq K k^{n} D(x, y)
$$

for any $x, y \in X$ and $n \geq 0$. Hence $\operatorname{Lip}\left(T^{n}\right) \leq K k^{n}$, for any $n \geq 0$. Therefore $\sum_{n \geq 0} \operatorname{Lip}\left(T^{n}\right)$ is convergent, which implies $T$ has a unique fixed point $\omega$, and any orbit converges to $\omega$.

From the definition of $D$ in the above Corollary, we easily see that $D$-convergence and $d$-convergence are identical.

Remark 3.5. In [1] the authors gave an example of a map $T$ which is contraction for $d$ but not for the euclidian distance. From the above corollary, we see that $\operatorname{Lip}(T) \leq K k$. Since $K k$ may not be less than 1 , then $T$ may not be a contraction for $D$. This is why the above theorems were stated in terms of $\left\{\operatorname{Lip}\left(T^{n}\right)\right\}$.

Using the ideas described above one can prove fixed point results for mappings which contracts orbits and obtain similar results as Theorem 4 for example in [1].

\section{References}

[1] L.-G. Huang and X. Zhang, "Cone metric spaces and fixed point theorems of contractive mappings," Journal of Mathematical Analysis and Applications, vol. 332, no. 2, pp. 1468-1476, 2007.

[2] B. Rzepecki, "On fixed point theorems of Maia type," Publications de l'Institut Mathématique, vol. 28(42), pp. 179-186, 1980.

[3] M. Abbas and G. Jungck, "Common fixed point results for noncommuting mappings without continuity in cone metric spaces," Journal of Mathematical Analysis and Applications, vol. 341, no. 1, pp. 416-420, 2008.

[4] D. Ilić and V. Rakočević, "Common fixed points for maps on cone metric space," Journal of Mathematical Analysis and Applications, vol. 341, no. 2, pp. 876-882, 2008.

[5] S. Rezapour and R. Hamlbarani, "Some notes on the paper: "Cone metric spaces and fixed point theorems of contractive mappings"," Journal of Mathematical Analysis and Applications, vol. 345, no. 2, pp. 719-724, 2008.

[6] D. Turkoglu and M. Abuloha, "Cone metric spaces and fixed point theorems in diametrically contractive mappings," Acta Mathematica Sinica, vol. 26, no. 3, pp. 489-496, 2010. 
[7] D. Turkoglu, M. Abuloha, and T. Abdeljawad, "KKM mappings in cone metric spaces and some fixed point theorems," Nonlinear Analysis: Theory, Methods \& Applications, vol. 72, no. 1, pp. 348-353, 2010.

[8] P. Vetro, "Common fixed points in cone metric spaces," Rendiconti del Circolo Matematico di Palermo. Serie II, vol. 56, no. 3, pp. 464-468, 2007.

[9] A. Quilliot, "An application of the Helly property to the partially ordered sets," Journal of Combinatorial Theory. Series A, vol. 35, no. 2, pp. 185-198, 1983.

[10] E. Jawhari, D. Misane, and M. Pouzet, "Retracts: graphs and ordered sets from the metric point of view," in Combinatorics and Ordered Sets (Arcata, Calif., 1985), vol. 57 of Contemporary Mathematics, pp. 175-226, American Mathematical Society, Providence, RI, USA, 1986.

[11] M. A. Khamsi and W. A. Kirk, An Introduction to Metric Spaces and Fixed Point Theory, Pure and Applied Mathematics, Wiley-Interscience, New York, NY, USA, 2001.

[12] M. A. Khamsi, W. M. Kozłowski, and S. Reich, "Fixed point theory in modular function spaces," Nonlinear Analysis: Theory, Methods E Applications, vol. 14, no. 11, pp. 935-953, 1990. 